\title{
CETIOM Stratégie plein champ : Oléopro 2002
}

\section{Cetiom Oleopro 2002, a strategy in the country}

Oléagineux, Corps Gras, Lipides. Volume 9, Numéro 4, 212-5, Juillet - Août 2002, La filière

Auteur(s) : Bernard SOUM, Florence DOAT-MATROT, Chef du département Éditions et communication du CETIOM, 12, avenue George-V, 75008 Paris.

Author(s) : Bernard SOUM, Florence DOAT-MATROT

Résumé : Pour la filière oléoprotéagineuse française, Oléopro 2002 aura été l'événement médiatique marquant de l'année 2002, le " premier salon au champ des oléoprotéagineux ". II s'est déroulé les 12 et 13 juin 2002 près de Bourges et a réuni 18000 visiteurs venus de toute la France, principalement des producteurs. À l'échelle de la filière oléoprotéagineuse, c'est un succès puisque $90 \%$ des visiteurs indiquent que la visite a répondu à leur attente, et que $98 \%$ des exposants se disent satisfaits des contacts réalisés. Le concept qui a conduit à ce succès est apparemment simple : présenter sur 40 hectares et $4000 \mathrm{~m} 2$ de tentes toutes les offres de progrès susceptibles d'intéresser les producteurs d'oléagineux et de protéagineux, de façon démonstrative et conviviale, le tout au grand air. Offres techniques d'une part, à travers 4 ha de micro-parcelles de démonstration mises en place par le Cetiom et I'UNIP 2-ITCF 3. Offres technico-commerciales d'autre part avec la présence de 56 sociétés exposantes, représentant aussi bien l'amont que l'aval de la production, ainsi que les organisations professionnelles du secteur.

Summary : In June 2002, Cetiom organized in France a huge informative field meeting for the French farmers: Oleopro 2002. It gathered 18,000 people and was considered highly useful and educative by the visitors. Three strategic concepts were at the basis of this event and explain its success: to show practicable innovations and operational issues, to develop interactive relationship with all the farmers' partners, and to be attentive to the emergent needs of the society. The author explains how the same trends are now leading the applied research program of Cetiom.

Keywords : Cetiom, popularization, field exhibition, innovation, strategy, applied research, Oleopro 2002.

\section{ARTICLE}

\section{Une stratégie de filière}

En réalité, la conception d'Oléopro 2002 est le résultat d'orientations qui trouvent leur inspiration dans la stratégie même de la filière Proléa. Celle-ci ne se limite pas à la conception d'Oléopro 2002. Elle a également inspiré le Cetiom, qui est l'organisme technique de la filière en charge de la recherche appliquée, de l'innovation et de son développement, dans la réforme récente de son organisation. En 2001 le centre a en effet été amené à modifier ses structures, à développer de nouveaux champs de compétences et à infléchir ses programmes. Il est intéressant de voir comment 
ces mêmes lignes directrices ont pu trouver leurs applications dans un événement ponctuel comme Oléopro 2002 et dans les orientations à moyen terme du Cetiom.

Cette stratégie s'exprime à travers trois ambitions. La première est de s'afficher comme force de proposition, sur la base de solutions innovantes et de technologies modernes, afin d'être un acteur efficace du changement. La seconde est de développer des relations interactives avec tous les acteurs de la production. La troisième est un parti pris d'écoute attentive des besoins de l'ensemble de la société.

\section{0 thèmes phare pour présenter Oléopro 2002}

À partir de novembre 2001, dix messages publicitaires ont été diffusés dans la presse agricole pour annoncer Oléopro 2002. Chacun traitait d'un thème précis, résumé d'une phrase, sur fond de portrait noir et blanc d'un agriculteur ( 7 fois), d'une agricultrice ( 1 fois), d'une diététicienne (1 fois) ou d'un cuisinier (1 fois). À chaque fois le nom était cité et la personne photographiée était également interviewée pour le spectacle audiovisuel présenté à Oléopro 2002. Suivaient quelques explications destinées à expliciter le contenu technique du message.

Ces dix messages ont ensuite assuré la cohérence de la communication pendant toute la préparation d'Oléopro 2002.

"Le colza, c'est un peu la vitamine de mon blé. »

« L'engrais que je donne à ma culture de pois? L'air ambiant !»

-- « La culture du tournesol a de quoi ensoleiller ma vie. »

"Qu'est-ce que je gagne à cultiver de la féverole ? De la liberté, et ça, ça n'a pas de prix. »

« Avec le soja de pays, on sait ce qu'on donne à manger aux animaux. Donc on sait ce qu'on mange. »

"Cultiver du colza, ça revient à fabriquer d'excellentes éponges. »

" On peut cuisiner sans huile. Oui. Comme on peut vivre sans rire et sans soleil. »

« Les oléopros, c'est bon pour la santé des moteurs. Et de la planète. »

«Avec les oléopros, j'ai parfois la sensation d'être un peintre. »

« Les oléagineux évitent de nombreux accidents de la circulation. »

"Venez voir pousser l'avenir " : telle était l'accroche retenue par la filière oléoprotéagineuse Proléa pour annoncer le salon au champ Oléopro 2002. À travers cette invitation, c'est une véritable stratégie d'action qui s'exprime, la même qui inspire le Cetiom ${ }^{1}$, principale cheville ouvrière de cet événement, dans son organisation et son fonctionnement quotidien. Analyse croisée de deux stratégies en marche. 


\section{Être une force de proposition réaliste}

Dans le cas d'Oléopro 2002, la volonté de s'afficher comme force de proposition s'est traduite par le cahier des charges qui a été proposé à tous les organisateurs, qu'ils soient centre technique ou société commerciale. II indiquait que toutes les propositions présentées à Oléopro 2002 devaient être des propositions de progrès directement accessibles au producteur agricole et, si possible, immédiatement opérationnelles. "Notre objectif est de présenter des informations très pratiques pour permettre aux producteurs de s'approprier de nouvelles techniques » indiquait Jacques Siret, président ce l'Onidol ${ }^{4}$ et président de l'association organisatrice d'Oléopro 2002. Et il ajoutait : " Depuis 10 ans, les cultures oléoprotéagineuses ont perdu 200 euros/ha de compétitivité [...]. S'ils peuvent bénéficier de 10 petites innovations techniques permettant de gagner chacune 20 euros/ha, ce déficit sera réduit. »

De fait l'information opérationnelle était abondante à Oléopro 2002. Dans le Village technique animé par le Cetiom et I'UNIP-ITCF, elle se présentait sous la forme de démonstrations (600 parcelles représentant 16 espèces et 300 variétés différentes), de posters explicatifs (68 thèmes présentés par 300 posters) et de visites commentées par les experts techniques du secteur (300 exposés ayant permis de toucher 4000 personnes, sur la base de 3 exposés écoutés par personne en moyenne), sans compter les réponses aux questions individuelles apportées par les spécialistes techniques présents. Sur les stands commerciaux, l'information était également focalisée sur les problématiques, les produits et les débouchés spécifiquement associés aux oléoprotéagineux, qu'il s'agisse de variétés, de produits phytosanitaires, de produits alimentaires transformés ou de débouchés industriels. Avec, à chaque fois, le parti-pris de donner à voir et de démontrer (photo 1).

Cette approche a été appréciée par les visiteurs puisque, dans l'enquête de satisfaction réalisée pendant l'événement, $92 \%$ se disent satisfaits de la présentation des thèmes et des démonstrations sur le Village technique. Et $85 \%$ sont satisfaits des commentaires qui leur ont été présentés. Les résultats sont similaires pour les informations et les commentaires dispensés par les stands commerciaux, ainsi que sur la disponibilité des exposants. De leur côté, les exposants ont été quasi unanimes à qualifier les visiteurs de « professionnels, exigeants, sérieux et motivés ».

\section{Orientation client}

Au niveau du Cetiom, cette exigence d'écoute et d'opérationnalité est une priorité. Elle a un nom : développer " l'orientation client ». Le but est de concevoir, de mettre au point et de faire connaître le maximum d'innovations utiles aux acteurs du secteur oléagineux. Il est également de faire en sorte que les innovations produites soient immédiatement utilisables par ceux à qui elles sont destinées. Cela se traduit par le fait que la plupart des programmes menés sont maintenant gérés comme des projets, avec des moyens propres et surtout des objectifs de production précis et fixés dans le temps. Une nouvelle fonction a été créée pour instruire, suivre et évaluer les projets en question : la fonction « d'animateur programmation, projets et évaluation ».

Cela se répercute également sur le choix des thématiques privilégiées. Ainsi les programmes visant à mettre au point des systèmes d'aide à la décision se développent, qu'ils s'intègrent dans des démarches tracées ou, plus simplement, dans la mise au point de " bonnes pratiques " adaptées aux exigences économiques et environnementales actuelles. Les préoccupations liées aux systèmes de culture et à la place des oléagineux dans ces systèmes se développent également. En effet, la 
demande des producteurs est, de plus en plus, d'optimiser les systèmes de culture au sein de l'exploitation et, de moins en moins, d'appliquer sans discernement des innovations venues de l'extérieur.

Concrètement, la structure du secteur recherche du Cetiom a été réformée de façon à se centrer sur les thématiques clé et non sur les seules technologies de production comme auparavant. Ainsi quatre services regroupent l'ensemble des spécialistes du Cetiom : innovations, systèmes de culture, valorisation des produits et économie/prospective. Ils apportent leur compétence à la mise en œuvre des différents projets, qui sont souvent pluridisciplinaires. Et ils encadrent les programmes de recherche, qu'ils soient conduits directement par le Cetiom ou menés par d'autres organismes (photo 2).

\section{Jouer gagnant-gagnant}

La seconde ambition qui a guidé la conception d'Oléopro 2002 est de développer des relations interactives avec les acteurs de la production. Elle s'est concrétisée, pendant les deux jours de l'événement, par la mobilisation de tous les collaborateurs de la filière pour animer les pôles présentés, et surtout pour répondre aux nombreuses questions posées par les visiteurs. Au total, 200 personnes du CETIOM, de I'ONIDOL et de I'UNIP/ITCF ont été mobilisées.

Auparavant la campagne de communication d'Oléopro 2002 avait décliné, pendant plusieurs mois, dix grands thèmes phares (voir encadré) présentant de façon imagée les informations qui seraient disponibles à Oléopro 2002. À l'issue de leur visite, $79 \%$ des visiteurs indiquaient qu'ils avaient en arrivant une question à poser, et $94 \%$ d'entre eux affirment y avoir trouvé réponse.

Mais le souci d'écoute et d'interactivité s'est surtout manifesté pendant la phase de préparation d'Oléopro 2002. Toutes les sociétés susceptibles d'être présentes ont été contactées individuellement, avec une démarche spécifique pour les sociétés commerciales d'amont (semenciers et produits phytosanitaires), une autre pour les sociétés commerciales d'aval (transformation, débouchés) et une autre pour les groupes de presse. Parallèlement les coopératives, négociants, chambres d'agriculture et groupes de développement susceptibles d'organiser des groupes de visite d'agriculteurs étaient contactés par les ingénieurs régionaux du Cetiom. L'objectif était de recenser les besoins de ces différents partenaires afin de parvenir à des propositions qui puissent s'intégrer dans leur propre politique. En même temps le but était de les inciter à adapter leurs projets pour assurer une cohérence d'ensemble et une synergie des différentes initiatives. Pratiquement tous les partenaires en question se sont dits satisfaits de cette méthode " gagnant-gagnant » qui a abouti à faire bénéficier chacun du dynamisme de l'ensemble, moyennant une acceptation au départ du cahier des charges proposé.

\section{Un rôle de médiateur actif}

Le Cetiom est en fait assez familier de cette démarche de " médiation active », dans le domaine de la recherche appliquée. II collabore directement avec de nombreux partenaires scientifiques, soit pour les alimenter en problématiques de recherche, soit pour collaborer avec eux sur des sujets communs, soit pour participer financièrement à leurs programmes scientifiques, par des contrats ou la prise en charge de thèses. Par exemple, sur le problème du phoma du colza, il collabore avec le service de la 
protection des végétaux dans un réseau de surveillance et d'avertissements, avec l'Inra ${ }^{5}$ de Versailles et de Rennes sur les aspects de sélection et de pathologie et finance une thèse d'agronomie à l'Inra de Grignon. S'ajoutent à cela des collaborations avec le Geves ${ }^{6}$ et les semenciers pour étudier l'adaptation des différentes variétés aux différentes souches de parasite.

Cela suppose d'avoir au sein même du Cetiom des chercheurs avec leurs propres programmes de recherche car c'est une condition indispensable pour être crédible et pouvoir dialoguer efficacement avec d'autres chercheurs. C'est pourquoi le Cetiom renforce, dans son organisation actuelle, ses laboratoires d'analyses, de pathologie et de biologie moléculaire, qui sont les supports de sa recherche avancée. Cette fonction d'interface entre recherche et acteurs économiques n'est d'ailleurs pas la seule où le Cetiom intervient. II s'attache également à assurer un rôle d'interface entre filière et territoire, ainsi qu'entre pouvoirs publics et profession agricole.

Mais il faut aujourd'hui aller plus loin que ces collaborations scientifiques classiques. En matière d'innovation il apparaît souhaitable d'associer les usagers de l'innovation à la création et à la mise au point des innovations elles-mêmes. Et pour cela mettre en route des processus de " co-construction " où agriculteurs et techniciens travaillent ensemble à identifier et construire des innovations dans le cadre de réseaux. Au Cetiom ces réseaux ont commencé à se mettre en place. Ils présentent des dimensions régionales très marquées car les problématiques liées à la gestion de l'exploitation, aux spécificités régionales et à la prise en compte des préoccupations liées au territoire sont essentielles. Au plan de l'organisation, cela s'est traduit par la création de nouvelles fonctions au sein des régions, pour aborder ces questions et, plus généralement animer des réseaux régionaux.

\section{À l'écoute de la société}

La troisième ambition qui a orienté Oléopro 2002 est d'afficher une volonté d'écoute des besoins de la société, et de démontrer que ces besoins ne représentent pas forcément des contraintes pour le secteur oléoprotéagineux mais peuvent aussi être des opportunités. Ainsi parmi les dix thèmes phare qui ont servi à annoncer l'événement dans la presse, quatre concernaient directement des préoccupations sociétales : l'intérêt nutritionnel des huiles végétales, le biocarburant et son bon comportement vis-à-vis de l'effet de serre, et l'utilité de rotations diversifiées pour le paysage. Et trois avaient trait à des préoccupations environnementales : l'intérêt des légumineuses du fait de leur non-consommation d'engrais azotés, les atouts du colza comme pompe à nitrates, et l'avantage de filières de soja tracées pour l'alimentation animale. L'objectif était, à chaque fois, de mettre en évidence une formulation du progrès technique compatible avec les besoins du consommateur ou du citoyen. Pendant les deux jours d'Oléopro 2002, ces dix thèmes ont été présentés aux visiteurs sous la forme d'un animation audio-visuelle spectaculaire, sous chapiteau, sur un écran géant de $26 \mathrm{~m}$ de long. L'objectif était de promouvoir les nombreuses raisons positives de développer les productions oléoprotéagineuses, et de le dire dans un langage " grand public " afin de signifier aux agriculteurs visiteurs que ces productions sont également porteuses d'espoir pour l'ensemble de la société. L'accueil de ce spectacle a été mitigé puisque $45 \%$ seulement l'ont vraiment apprécié. II faut dire qu'il avait été présenté dans des conditions matérielles très défavorables : obligation de parcourir plusieurs kilomètres pour le voir suite à un accident climatique ayant empêché l'installation du chapiteau sur le site même d'Oléopro 2002. Mais il a probablement participé à éveiller les visiteurs aux enjeux, pour l'agriculture, des évolutions actuelles de la société. 
Il faut également signaler une initiative intéressante sur Oléopro 2002 : la mise en place d'un circuit de visite spécifiquement dédié aux citadins et ruraux non agriculteurs de la région, reprenant quelques thèmes techniques de l'événement dans une présentation accessible au grand public. Dix posters, répartis sur l'ensemble du site ont permis d'aborder les principales problématiques sociétales concernant les oléoprotéagineux. Commentés par des agriculteurs de la région, ils ont été en fait peu valorisés du fait de la très faible fréquentation de citadins. S'il est difficile d'en tirer des leçons vis-à-vis du public des citadins, il apparaît néanmoins qu'ils ont été appréciés des agriculteurs visiteurs (photo 4).

\section{Communication interactive}

Cette préoccupation d'écoute et d'anticipation des besoins de la société s'est traduite au CETIOM par la mise en place d'une unité de Prospective qui se donne pour mission de recueillir les éléments et les analyses susceptibles d'éclairer les décisions de tous les acteurs du secteur, et pas seulement les producteurs. Elle se retrouve également dans l'orientation des programmes qui, à côté des actions visant à améliorer la compétitivité économique, s'attachent de plus en plus à prendre en compte les préoccupations environnementales et de sécurité sanitaire. Plusieurs unités ont de ce fait été renforcées par des compétences nouvelles, en particulier dans les domaines des systèmes de culture, du diagnostic environnemental, de l'agriculture de précision et de la sécurité alimentaire.

Pour être partie prenante des évolutions du secteur oléoprotéagineux, le Cetiom affiche sa volonté d'être " acteur parmi les acteurs ». Ceci implique des relations permanentes et interactives avec tous ses partenaires, dans lesquelles il est probable que les nouvelles technologies de l'information joueront un rôle important. De même qu'Oléopro 2002 a été, pour la filière Proléa, l'occasion ponctuelle de communiquer à la fois avec les agriculteurs, les opérateurs économiques, les médias et le grand public, de même l'ambition du Cetiom est, pour les années à venir, d'utiliser les nouvelles technologies de l'information comme support permanent de relations interactives avec les producteurs, les techniciens-prescripteurs, le monde scientifique et les relais d'opinion.

Notes:

${ }^{1}$ Cetiom : Centre technique interprofessionnel des oléagineux métropolitains.

${ }^{2}$ UNIP : Union nationale interprofessionnelle des plantes riches en protéines.

${ }^{3}$ ITCF : Institut technique de céréales et des fourrages.

${ }^{4}$ Onidol : Organisation nationale interprofessionnelle des oléagineux.

${ }^{5}$ Inra : Institut national de la recherche agronomique.

${ }^{6}$ Geves : Groupe d'étude et de contrôle des variétés et des semences. 


\section{Illustrations}

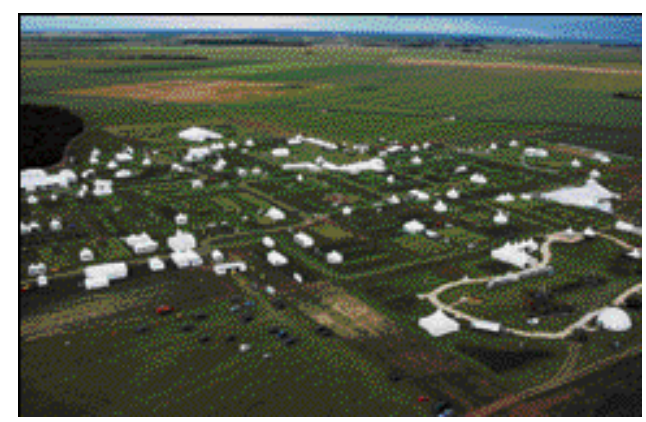

Photo 1. Oléopro 2002 : 40 ha d'information, une véritable ville avec ses rues, ses restaurants, ses maisons.

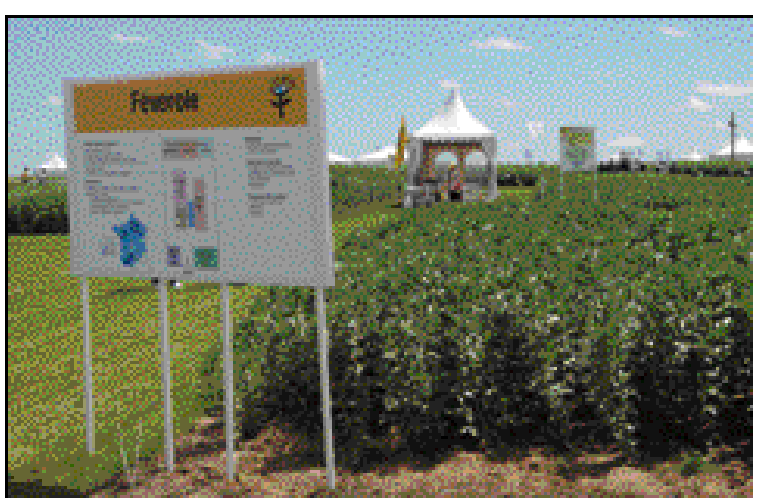

Photo 2. Au sein du Village technique, les tentes d'information viennent compléter les démonstrations sur les parcelles.

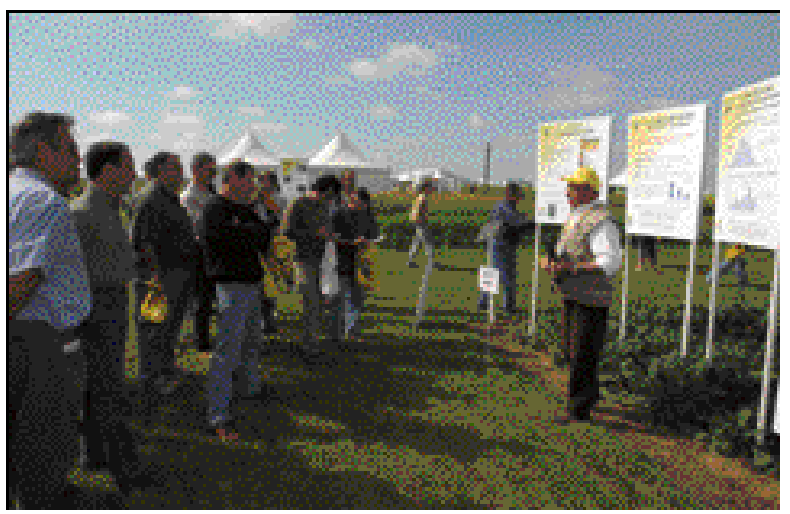

Photo 3. Chaque thème est commenté par un spécialiste et appuyé par des posters. 


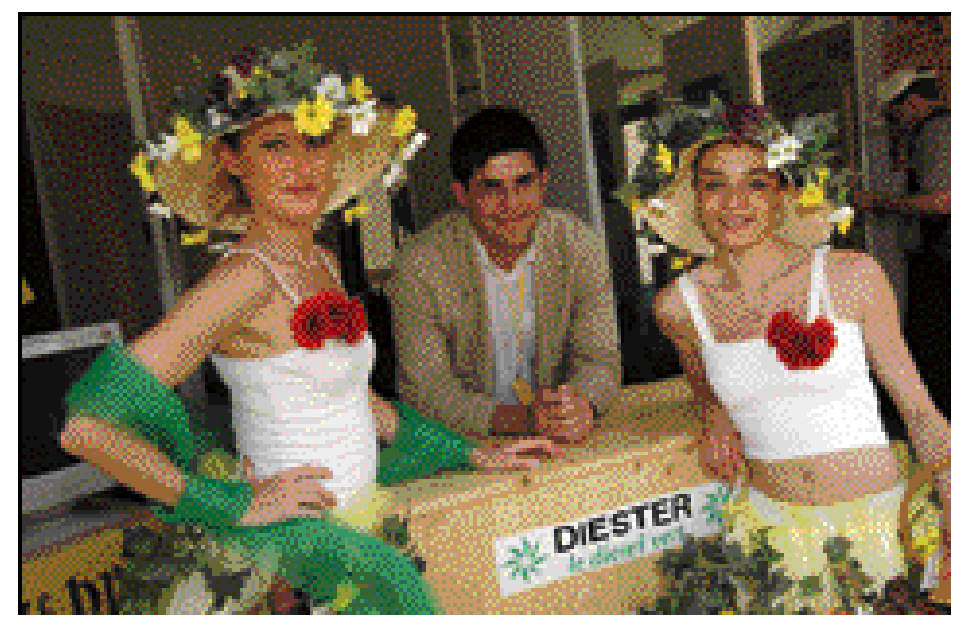

Photo 4. La promotion du biocarburant Diester n'exclut pas la convivialité et la recherche esthétique. 